\title{
INTERPOLATION OPERATORS ON A TRIANGLE WITH ALL CURVED SIDES
}

\author{
Alina BABOȘ \\ "Nicolae Bălcescu" Land Forces Academy, Sibiu, Romania \\ alina_babos_24@yahoo.com
}

\begin{abstract}
This paper contains a survey regarding interpolation and Cheney-Sharma type operators defined on a triangle with all curved sides; we considers as well some of the product and Boolean sum operators. We study their interpolation properties and the degree of exactness.
\end{abstract}

Keywords: interpolation operators, triangle with curved sides, Cheney-Sharma type operators, products and Boolean sum operators

\section{Introduction}

The aim of this survey is to present some interpolation and Cheney-Sharma type operators for functions defined on a triangle with all curved side (see [1], [2]). They came as an extension of the corresponding operators for functions defined on triangles with all straight sides (see, e.g., [3], [4], [9] ).

We study these operators especially from the theoretical point of view.

We study two main aspects of the constructed operators: the interpolation properties and the degree of exactness.

Recall that $\operatorname{dex}(\mathrm{P})=r$ (where $\mathrm{P}$ is an interpolation operator) if $\mathrm{P} f=f$, for $f \in P_{r}$, and there exists $g \in P_{r+1}$ such that $P g \neq g$, where $P_{m}$ denote the space of the polynomials in two variables of global degree at most $m$.
In Section 2 we study Lagrange, Hermite and Birkhoff interpolation operators and in Section 3 we present some Cheney-Sharma type operators together with their product and Boolean sum for the triangle with all curved sides.

Given $h>0$, denote by $\widetilde{T}_{h}$ the triangle having the vertices $V_{1}=(0, h), V_{2}=(h, 0)$ and $V_{3}=(0,0)$, and the three curved sides $\gamma_{1}, \gamma_{2}$ (along the coordinates axes) and $\gamma_{3}$ (opposite to the vertex $V_{3}$ ). We define $\gamma_{1}$ by $\left(x, f_{1}(x)\right)$ with $f_{1}(0)=f_{1}(h)=0, f_{1}(x) \leq 0$, for $x \in[0, h] ; \gamma_{2}$ defined by $\left(g_{2}(y), y\right)$, with $g_{2}(0)=g_{2}(h)=0, g_{2}(y) \leq 0$, for $y \in[0, h]$ and $\gamma_{3}$ defined by one-to-one functions $f_{3}$ and $g_{3}$, where $g_{3}$ is the inverse of the function $f_{3}$, i.e., $y=f_{3}(x)$ and $x=g_{3}(y)$, with $x, y \in[0, h]$ and $f_{3}(0)=g_{3}(0)=h$, $h \in R_{+}$, (see Figure 1). 


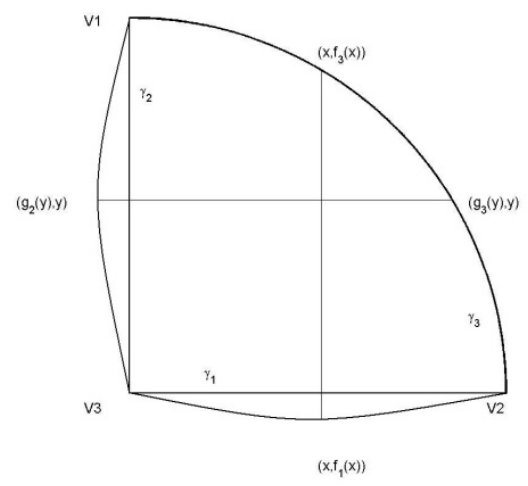

Figure 1. Triangle $\widetilde{T}_{h}$

\section{Interpolation operators}

\subsection{Lagrange-type operators}

Suppose that $F$ is a real-valued function defined on $\widetilde{T}_{h}$. Let $L_{1}$ and $L_{2}$ be the interpolation operators defined by

$$
\begin{aligned}
\left(L_{1} F\right)(x, y) & =\frac{x-g_{3}(y)}{g_{2}(y)-g_{3}(y)} F\left(g_{2}(y), y\right) \\
& +\frac{x-g_{2}(y)}{g_{3}(y)-g_{2}(y)} F\left(g_{3}(y), y\right)
\end{aligned}
$$$$
\left(L_{2} F\right)(x, y)=\frac{y-f_{3}(x)}{f_{1}(x)-f_{3}(x)} F\left(x, f_{1}(x)\right)
$$$$
+\frac{y-f_{1}(x)}{f_{3}(x)-f_{1}(x)} F\left(x, f_{3}(x)\right)
$$

Theorem 1.([1]) If $F: \widetilde{T}_{h} \rightarrow R$, then we get (1) the interpolation properties: $L_{1} F=F$, on $\gamma_{2} \cup \gamma_{3}, L_{2} F=F$, on $\gamma_{1} \cup \gamma_{3}$.

(2) the degree of exactness: $\operatorname{dex}\left(L_{i}\right)=1$, $i=\overline{1,2}$.

Proof. (1) $\left(L_{1} F\right)\left(g_{2}(y), y\right)=F\left(g_{2}(y), y\right)$, $\left(L_{1} F\right)\left(g_{3}(y), y\right)=F\left(g_{3}(y), y\right)$, $\left(L_{2} F\right)\left(x, f_{1}(x)\right)=F\left(x, f_{1}(x)\right)$,
$\left(L_{2} F\right)\left(x, f_{3}(x)\right)=F\left(x, f_{3}(x)\right)$.

So, the interpolation properties are verified.

(2) $L_{1} e_{i j}=e_{i j}$ for $i, j \in N, i, j \leq 1$ and

$L_{1} e_{20} \neq e_{20}$, where $e_{i j}(x, y)=x^{i} y^{j}$. So. It follows that $\operatorname{dex}\left(L_{i}\right)=1$.

\subsection{Hermite-type operators}

Suppose that $F$ has the partial derivatives $F^{(1,0)}$ on $\gamma_{2}$ and $\gamma_{3}$ respectively $F^{(0,1)}$ on $\gamma_{1}$ and $\gamma_{3}$. We consider the operators $H_{1}$ and $\mathrm{H}_{2}$ defined by

$$
\begin{aligned}
& \left(H_{1} F\right)(x, y)=\frac{\left[x-g_{3}(y)\right]^{2}\left[3 g_{2}(y)-g_{3}(y)-2 x\right]}{\left[g_{2}(y)-g_{3}(y)\right]^{3}} . \\
& F\left(g_{2}(y), y\right)+\frac{\left[x-g_{2}(y)\right]^{2}\left[3 g_{3}(y)-g_{2}(y)-2 x\right]}{\left[g_{3}(y)-g_{2}(y)\right]^{3}} .
\end{aligned}
$$$$
F\left(g_{3}(y), y\right)+\frac{\left[x-g_{2}(y)\right]\left[x-g_{3}(y)\right]^{2}}{\left[g_{2}(y)-g_{3}(y)\right]^{2}} \text {. }
$$$$
F^{(1,0)}\left(g_{2}(y), y\right)+\frac{\left[x-g_{3}(y)\right]\left[x-g_{2}(y)\right]^{2}}{\left[g_{3}(y)-g_{2}(y)\right]^{2}} \text {. }
$$

$F^{(1,0)}\left(g_{3}(y), y\right)$

and 


$$
\begin{aligned}
& \left(H_{2} F\right)(x, y)=\frac{\left[y-f_{3}(x)\right]^{2}\left[3 f_{1}(x)-f_{3}(x)-2 y\right]}{\left[f_{1}(x)-f_{3}(x)\right]^{3}} . \\
& F\left(x, f_{1}(x)\right)+\frac{\left[y-f_{1}(x)\right]^{2}\left[3 f_{3}(x)-f_{1}(x)-2 y\right]}{\left[f_{3}(x)-f_{1}(x)\right]^{3}} \\
& F\left(x, f_{3}(x)\right)+\frac{\left[y-f_{1}(x)\right]\left[y-f_{3}(x)\right]^{2}}{\left[f_{1}(x)-f_{3}(x)\right]^{2}} . \\
& F^{(0,1)}\left(x, f_{1}(x)\right)+\frac{\left[y-f_{3}(x)\right]\left[y-f_{1}(x)\right]^{2}}{\left[f_{3}(x)-f_{1}(x)\right]^{2}} \\
& F^{(0,1)}\left(x, f_{3}(x)\right)
\end{aligned}
$$

Theorem 2.([1]) If $F: \widetilde{T}_{h} \rightarrow R$, then we get (1) the interpolation properties: $H_{1} F=F$, on $\gamma_{2} \cup \gamma_{3}, H_{1} F^{(1,0)}=F^{(1,0)}$, on $\gamma_{2} \cup \gamma_{3}$, $H_{2} F=F$, on $\gamma_{1} \cup \gamma_{3}, H_{2} F^{(0,1)}=F^{(0,1)}$, on $\gamma_{1} \cup \gamma_{3}$.

(2) the degree of exactness:

$$
\operatorname{dex}\left(H_{1}\right)=\operatorname{dex}\left(H_{2}\right)=2 \text {. }
$$

Proof. (1) $\left(H_{1} F\right)\left(g_{2}(y), y\right)=F\left(g_{2}(y), y\right)$,

$\left(H_{1} F\right)\left(g_{3}(y), y\right)=F\left(g_{3}(y), y\right)$,

$\left(H_{1} F\right)^{(1,0)}(x, y)=$

$\frac{6\left[x-g_{3}(y)\right]\left[g_{2}(y)-x\right]}{\left[g_{2}(y)-g_{3}(y)\right]^{3}} \cdot F\left(g_{2}(y), y\right)$

$+\frac{6\left[x-g_{2}(y)\right]\left[g_{3}(y)-x\right]}{\left[g_{3}(y)-g_{2}(y)\right]^{3}} \cdot F\left(g_{3}(y), y\right)$

$+\frac{\left[x-g_{3}(y)\right]\left[3 x-2 g_{2}(y)-g_{3}(y)\right]}{\left[g_{2}(y)-g_{3}(y)\right]^{2}}$.

$F^{(1,0)}\left(g_{2}(y), y\right)$

$+\frac{\left[x-g_{2}(y)\right]\left[3 x-2 g_{3}(y)-g_{2}(y)\right]}{\left[g_{3}(y)-g_{2}(y)\right]^{2}}$.

$F^{(1,0)}\left(g_{3}(y), y\right)$.

We have:

$\left(H_{1} F\right)^{(1,0)}\left(g_{2}(y), y\right)=F^{(1,0)}\left(g_{2}(y), y\right)$,

$\left(H_{1} F\right)^{(1,0)}\left(g_{3}(y), y\right)=F^{(1,0)}\left(g_{3}(y), y\right)$.

Also for the interpolation properties of $\mathrm{H}_{2}$.

So, it follows that $\operatorname{dex}\left(B_{1}\right)=1$. Similar for $\operatorname{dex}\left(B_{2}\right)=1$.
(2) We obtain $H_{1} e_{i j}=e_{i j}$ for $i, j<2$ and $H_{1} e_{30} \neq e_{30}$, where $e_{i j}(x, y)=x^{i} y^{j}$. So, it follows that $\operatorname{dex}\left(H_{1}\right)=2$. Similar for $\operatorname{dex}\left(H_{2}\right)=2$.

\subsection{Birkhoff-type operators}

We give some examples of operators which interpolate the given function $F: \widetilde{T}_{h} \rightarrow R$ on a side of triangle, respectively, its partial derivatives on the others side.

We suppose that the function $F: \widetilde{T}_{h} \rightarrow R$ has the partial derivatives $F^{(0,1)}$ on $\gamma_{3}$ and $\gamma_{1}$ and $F^{(1,0)}$ on $\gamma_{3}$.

We consider the Birkhoff-type operators $B_{1}$ and $B_{2}$ defined by

$$
\begin{aligned}
\left(B_{1} F\right)(x, y) & =F\left(g_{2}(y), y\right) \\
+ & \left(x-g_{2}(y)\right) F^{(1,0)}\left(g_{3}(y), y\right) \\
\left(B_{2} F\right)(x, y) & =F\left(x, f_{1}(x)\right) \\
+ & \left(y-f_{1}(x)\right) F^{(0,1)}\left(x, f_{3}(x)\right)
\end{aligned}
$$

Theorem 3.([1]) If $F: \widetilde{T}_{h} \rightarrow R$, then we get (1) the interpolation properties: $B_{1} F=F$ on $\gamma_{2},\left(B_{1} F\right)^{(1,0)}=F^{(1,0)}$ on $\gamma_{3}, B_{2} F=F$ on $\gamma_{1}$, $\left(B_{2} F\right)^{(0,1)}=F^{(0,1)}$ on $\gamma_{3}$.

(2) the degree of exactness: $\operatorname{dex}\left(B_{1}\right)=\operatorname{dex}\left(B_{2}\right)=1$.

Proof. (1) $\left(B_{1} F\right)\left(g_{2}(y), y\right)=F\left(g_{2}(y), y\right)$, $\left(B_{1} F\right)^{(1,0)}\left(g_{3}(y), y\right)=F^{(1,0)}\left(g_{3}(y), y\right)$, $\left(B_{1} F\right)\left(x, f_{1}(x)\right)=F\left(x, f_{1}(x)\right)$, $\left(B_{1} F\right)^{(0,1)}\left(x, f_{3}(x)\right)=F^{(0,1)}\left(x, f_{3}(x)\right)$.

(2) $B_{1} e_{i j}=e_{i j}$ for $i, j \leq 1$ and $B_{1} e_{20} \neq e_{20}$, where $e_{i j}(x, y)=x^{i} y^{j}$.

\section{Cheney-Sharma type operators}

The Cheney-Sharma type operators on a triangle with curved sides are extension of the Cheney-Sharma type operators of 
second type, given by E.W.Cheney and A.Sharma in [6].

Let $m \in N$ and $\beta$ a nonnegative parameter. The Cheney-Sharma operators of second kind $Q_{m}: C([0,1]) \rightarrow C([0,1])$, introduced in [6], are given by

$$
\left(Q_{m} f\right)(x)=\sum_{i=0}^{m} q_{m, i}(x) f\left(\frac{i}{m}\right)
$$

$q_{m, j}(x)=$

$\left(\begin{array}{c}m \\ i\end{array}\right) \frac{x(x+i \beta)^{i-1}(1-x)[1-x+(m-i) \beta]^{m-i-1}}{(1+m \beta)^{m-1}}$

For $m, n \in N, \alpha, \beta \in R_{+}$, we consider the following extensions of the Cheney-Sharma operator given in (7):

Let $F$ be a real-valued function defined on $\widetilde{T}_{h}$ and $\left(x, f_{1}(x)\right),\left(x, f_{3}(x)\right)$, respectively, $\left(g_{2}(y), y\right),\left(g_{3}(y), y\right)$ the points in which the parallel lines to the coordinates axes, passing through the point $(x, y) \in \widetilde{T}_{h}$, intersect the sides $\gamma_{1}, \gamma_{2}$ and $\gamma_{3}$ (see Figure 1). We consider the uniform partitions of the intervals $\left[g_{2}(y), g_{3}(y)\right]$ and $\left[f_{1}(x), f_{3}(x)\right], x, y \in[0, h]:$

$\Delta_{m}^{x}=\left\{g_{2}(y)+i \frac{g_{3}(y)-g_{2}(y)}{m} \mid i=\overline{0, m}\right\}$,

respectively,
$\left(Q^{x} F\right)(x)=$

$\sum_{i=0}^{m} q_{m, i}(x, y) F\left(g_{2}(y)+i \frac{g_{3}(y)-g_{2}(y)}{m}, y\right)$,

$q_{m, i}(x, y)=\left(\begin{array}{c}m \\ i\end{array}\right) \frac{\frac{x-g_{2}(y)}{g_{3}(y)-g_{2}(y)}\left(\frac{x-g_{2}(y)}{g_{3}(y)-g_{2}(y)}+i \beta\right)}{(1+m \beta)^{m-1}}$.

$\left[1-\frac{x-g_{2}(y)}{g_{3}(y)-g_{2}(y)}\right] \cdot\left[1-\frac{x-g_{2}(y)}{g_{3}(y)-g_{2}(y)}+(m-i) \beta\right]^{m-i-1}$

respectively,

$\left(Q^{y}{ }_{n} F\right)(x)=$

$\sum_{j=0}^{n} q_{n, j}(x, y) F\left(x, f_{1}(x)+j \frac{f_{3}(x)-f_{1}(x)}{n}\right)$,

with

$\cdot\left[1-\frac{y-f_{1}(x)}{f_{3}(x)-f_{1}(x)}\right]\left[1-\frac{y-f_{1}(x)}{f_{3}(x)-f_{1}(x)}+(n-j) \alpha\right]^{n-j-1}$.

Theorem 4.([2]) If $F$ is a real-valued function defined on $\widetilde{T}_{h}$ then

(1) $Q_{m}^{x} F=F$ on $\gamma_{2} \cup \gamma_{3}$,

(2) $Q_{n}^{y} F=F$ on $\gamma_{1} \cup \gamma_{3}$,

(3) $\left(Q_{n}^{y} e_{i j}\right)(x, y)=x^{i} y^{j}, i=0,1 ; j \in N$,

(4) $\left(Q_{n}^{y} e_{i j}\right)(x, y)=x^{i} y^{j}, i \in N, j=0,1$.

Proof. 1) We write

$$
\begin{aligned}
& \Delta_{n}^{y}=\left\{f_{1}(x)+j \frac{f_{3}(x)-f_{1}(x)}{n} \mid j=\overline{0, n}\right\} . \\
& q_{n, j}(x, y)=\left(\begin{array}{c}
n \\
j
\end{array}\right) \frac{\frac{y-f_{1}(x)}{f_{3}(x)-f_{1}(x)}\left(\frac{y-f_{1}(x)}{f_{3}(x)-f_{1}(x)}+j \alpha\right)}{(1+n \alpha)^{n-1}}
\end{aligned}
$$




$$
\begin{aligned}
& \left(Q_{m}^{x} F\right)(x, y)= \\
& \frac{1}{(1+m \beta)^{m-1}}\left\{\left[1-\frac{x-g_{2}(y)}{g_{3}(y)-g_{2}(y)}\right] .\right. \\
& {\left[1-\frac{x-g_{2}(y)}{g_{3}(y)-g_{2}(y)}+m \beta\right]^{m-1} \cdot F\left(g_{2}(y), y\right)} \\
& +\frac{x-g_{2}(y)}{g_{3}(y)-g_{2}(y)}\left[1-\frac{x-g_{2}(y)}{g_{3}(y)-g_{2}(y)}\right] . \\
& \sum_{i=0}^{m}\left(\begin{array}{l}
m)\left(\frac{x-g_{2}(y)}{g_{3}(y)-g_{2}(y)}+i \beta\right)^{i-1} \cdot \\
{\left[1-\frac{x-g_{2}(y)}{g_{3}(y)-g_{2}(y)}+(m-i) \beta\right]^{m-i-1} .} \\
F\left(g_{2}(y)+i \frac{g_{3}(y)-g_{2}(y)}{m}, y\right) \\
+\frac{x-g_{2}(y)}{g_{3}(y)-g_{2}(y)} \cdot\left[\frac{x-g_{2}(y)}{g_{3}(y)-g_{2}(y)}+m \beta\right]^{m-1} . \\
\left.F\left(g_{3}(y), y\right)\right\}
\end{array} .\right.
\end{aligned}
$$

So,

$$
\begin{aligned}
& \left(Q_{m}^{x} F\right)\left(g_{2}(y), y\right)=F\left(g_{2}(y), y\right), \\
& \left(Q_{m}^{x} F\right)\left(g_{3}(y), y\right)=F\left(g_{3}(y), y\right) .
\end{aligned}
$$

The 2) is proved in a similar way with 1).

The proof for 3) and 4) follows by the property $\operatorname{dex}\left(Q_{m}\right)=1$ (proved in [6]).

Let $P_{m n}^{1}=Q_{m}^{x} Q_{n}^{y}$, respectively, $P_{n m}^{2}=Q_{n}^{y} Q_{m}^{x}$ be the product of the operators $Q_{m}^{x}$ and $Q_{n}^{y}$. We have

$$
\begin{aligned}
\left(P_{m n}^{1} F\right)(x, y) & =\sum_{i=0}^{m} \sum_{j=0}^{n} q_{m, i}(x, y) q_{n, j}\left(x_{i}, y\right) . \\
& F\left(x_{i}, f_{i}\left(x_{i}\right)+j \frac{f_{3}\left(x_{i}\right)-f_{1}\left(x_{i}\right)}{n}\right), \\
x_{i} & =g_{2}(y)+i \frac{g_{3}(y)-g_{2}(y)}{m},
\end{aligned}
$$

and

$$
\begin{gathered}
\left(P_{n m}^{2} F\right)(x, y)=\sum_{i=0}^{m} \sum_{j=0}^{n} q_{m, i}\left(x, y_{j}\right) q_{n, j}(x, y) \cdot \\
F\left(g_{2}\left(y_{j}\right)+i \frac{g_{3}\left(y_{j}\right)-g_{2}\left(y_{j}\right)}{m}, y_{j}\right), \\
y_{j}=f_{1}(y)+j \frac{f_{3}(x)-f_{1}(x)}{n} .
\end{gathered}
$$

Theorem 5.([2]) If $F$ is a real-valued function defined on $\widetilde{T}_{h}$ then:

1) $\left(P_{m n}^{1} F\right)\left(V_{3}\right)=F\left(V_{3}\right),\left(P_{m n}^{1} F\right)=F$, on $\Gamma_{3}$

2) $\left(P_{n m}^{2} F\right)\left(V_{3}\right)=F\left(V_{3}\right),\left(P_{n m}^{2} F\right)=F$, on $\Gamma_{3}$

Proof. The proof follows from the properties:

$$
\begin{aligned}
& \left(P_{m n}^{1} F\right)(x, 0)=\left(Q_{m}^{x} F\right)(x, 0), \\
& \left(P_{m n}^{1} F\right)(0, y)=\left(Q_{n}^{y} F\right)(0, y), \\
& \left(P_{m n}^{1} F\right)\left(x, f_{3}(x)\right)=F\left(x, f_{3}(x)\right), x, y \in[0, h]
\end{aligned}
$$

and

$$
\left(P_{n m}^{2} F\right)(x, 0)=\left(Q_{m}^{x} F\right)(x, 0),
$$

$\left(P_{n m}^{2} F\right)(0, y)=\left(Q_{n}^{y} F\right)(0, y)$,

$\left(P_{n m}^{2} F\right)\left(g_{3}(x), y\right)=F\left(g_{3}(y), y\right), x, y \in[0, h]$

which can be verified by a straightforward computation.

We consider the Boolean sums of the operators $Q_{m}^{x}$ and $Q_{n}^{y}$, i.e.,

$S_{m n}^{1}=Q_{m}^{x} \oplus Q_{n}^{y}=Q_{m}^{x}+Q_{n}^{y}-Q_{m}^{x} Q_{n}^{y}$,

respectively

$S_{n m}^{2}=Q_{n}^{y} \oplus Q_{m}^{x}=Q_{n}^{y}+Q_{m}^{x}-Q_{n}^{y} Q_{m}^{x}$

Theorem 6.([2]) If $F$ is a real-valued function defined on $\widetilde{T}_{h}$ then:

$$
\left.S_{m n}^{1}\right|_{\partial \widetilde{T}}=\left.F\right|_{\partial \widetilde{T}}
$$


$\left.S_{n m}^{2}\right|_{\partial \widetilde{T}}=\left.F\right|_{\partial \widetilde{T}}$

Proof.

As
$\left(S_{m n}^{1} F\right)\left(g_{2}(y), y\right)=\left(Q_{n}^{y} F\right)\left(g_{2}(y), y\right)$

$\left(S_{m n}^{1} F\right)\left(x, f_{3}(x)\right)=F\left(x, f_{3}(x)\right)$

the proof follows.

\section{References}

[1] Baboș, A., Interpolation operators on a triangle with two and three curved edges, Creat. Math. Inform., No. 2, pp. 135-142, 2011.

[2] Baboș, A., Cheney-Sharma type operators on a triangle with two and three curved edges, Ukrainian Mathematical Journal (accepted).

[3] Barnhill, R.E., Birkhoff, G., Gordon, W.J., Smooth interpolation in triangles, J. Approximation Theory, 8, pp. 114-128, 1973.

[4] Barnhill, R.E., Gregory, J.A., Polynomial interpolation to boundary data on triangles, Math. Comp., 29, pp. 726-735, 1975.

[5] Cătinaș, T., Blaga, P., Coman, Gh., Interpolation operators on some triangles with curved sides, Analele Științifice ale Universității "Al. I. Cuza" din Iași (S.N.) Matematică, Tomul LX, pp. 450-487, 2014.

[6] Cheney, E.W., Sharma, A., On a generalization on Bernstein polynomials, Analele Riv. Mat. Univ. Parma, 5, pp. 77-84, 1964

[7] Coman, Gh., Cătinaș, T., Interpolation operators on a triangle with one curved side, BIT, 50, pp. 243-267, 2010.

[8] Blaga, P., Cătinaș, T., Coman, Gh., Bernstein-type operators on a triangles with one curved side, Mediterr. J. Math., 9, pp. 833-845, 2012.

[9] Cătinaș, T., Extension of some positive and linear operators to domain with curved sides, MATEC Web of Conferences.,68, 16006, 2016.

[10] Gregory, J.A, A blending function interpolant for triangles, Multivariate Approximation, pp. 279-287, 1978. 